\title{
Integrating Healthcare Knowledge Artifacts for Clinical Decision Support: Towards Semantic Web Based Healthcare Knowledge Morphing
}

\author{
Sajjad Hussain and Syed Sibte Raza Abidi
}

NICHE Research Group, Faculty of Computer Science, Dahousie University, Canada

\begin{abstract}
Healthcare decision making demands the systematic integration of knowledge from multiple sources, such as clinical guidelines, clinical pathways, knowledge of practitioners and so on. We present a semantic web based approach for synthesizing health knowledge through the semantic modeling of healthcare knowledge as ontologies and reasoning over the ontologies to derive a morphed knowledge object. We demonstrate the application of our approach by generating morphed knowledge about prostate cancer clinical pathways.
\end{abstract}

\section{Introduction}

Healthcare decision making during the diagnostic-treatment cycle is a complex activity. Health professionals make clinical decisions by applying healthcare knowledge that includes their experiential knowledge and explicit knowledge 'artifacts', such as clinical practice guidelines, best evidence, clinical pathways and so on [1. One may note that each healthcare knowledge artifact captures specific conceptual, contextual and operational aspects of a disease and corresponding diagnostic/therapeutic procedures. Health professionals, guided by the patient's context, are able to select the relevant 'knowledge objects' from these different artifacts and then inter-relate these specific knowledge objects whilst satisfying clinical relevance and pragmatics constraints. For instance, a health professional generating a treatment plan for a patient with hypertension and diabetes will refer to the relevant sections of (a) clinical guidelines for recommendations; (b) clinical pathways for procedural protocols to exercise these recommendations; and (c) medical literature to determine the best evidence and outcomes of treatment options. In our work, we attempt to pursue a context-sensitive selection and integration of medical knowledge from multiple knowledge artifacts to generate a comprehensive knowledge object for clinical decision support.

We are developing the concept of healthcare knowledge morphing that entails "the intelligent and autonomous fusion/integration of contextually, conceptually and functionally related knowledge objects that may exist in different representation modalities and formalisms, in order to establish a comprehensive, multifaceted and networked view of all knowledge pertaining to a domain-specific problem"-Abidi 2005 [2]. In this paper, we present our healthcare knowledge

C. Combi, Y. Shahar, and A. Abu-Hanna (Eds.): AIME 2009, LNAI 5651, pp. 171-175 2009.

(C) Springer-Verlag Berlin Heidelberg 2009 


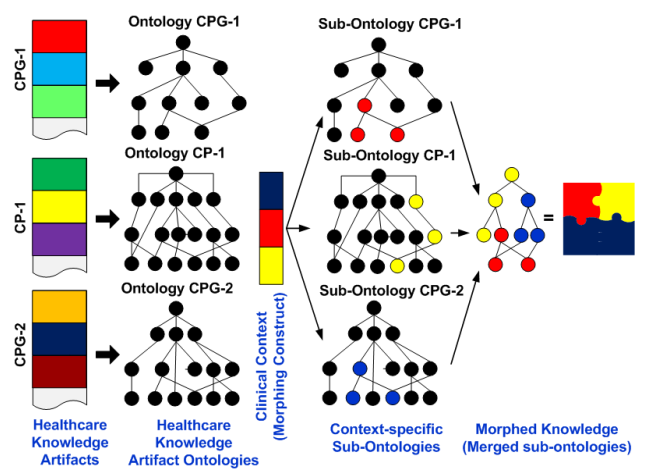

Fig. 1. Healthcare Knowledge Morphing

morphing framework $\mathcal{K}-\mathcal{M O} \mathcal{O} \mathcal{P} \mathcal{H}$ that is based on the semantic web approach that entails: (a) Developing Knowledge Artifact Ontologies (KAOs) to represent knowledge within CPG, CP and CM 11; (b) Specifying the clinical context of the knowledge morphing activity through a rich morphing construct; (c) Generating the morphed knowledge by (i) selecting a contextualized sub-ontology, corresponding to the clinical context, from the $\mathrm{KAO}$; and (ii) merging the selected contextualized sub-ontologies, using reasoning algorithms applied to a set of domain-specific context-specific axioms, to generate a new sub-ontology that represents the 'morphed' knowledge artifact. We demonstrate the working of our knowledge morphing framework $\mathcal{K}-\mathcal{M O} \mathcal{R} \mathcal{P H}$ by morphing three different location-specific clinical pathways to generate a comprehensive knowledge about treatments and follow-ups for a clinical context therapeutic decision support (see Figure 1) [1.

\section{Related Work}

The literature suggests other approaches for knowledge integration problem from different perspectives. For instance, the ECOIN framework performs semantic reconciliation of independent data sources, under a defined context, by defining conversion functions between contexts as a network. ECOIN takes the single ontology, multiple views approach [3, and introduces the notion of modifiers to explicitly describe the multiple specializations/views of the concepts used in different data sources. It exploits the modifiers and conversion functions, to enable context mediation between data sources, and reconcile and integrate source schemas with respect to their conceptual specializations. Another recent initiative towards knowledge integration is the OpenKnowledge project [4] that supports the knowledge sharing among different knowledge artifacts, not by sharing their asserted statements, instead by sharing their interaction models. An interaction model provides a context in which knowledge can be transmitted between two (or more) knowledge sources (peers). 


\section{$3 \quad \mathcal{K}-\mathcal{M O} \mathcal{R} \mathcal{P} \mathcal{H}$ Architecture}

The $\mathcal{K}-\mathcal{M O R P H}$ approach is shown in Figure 1, and its main elements are described in the following subsections. For further details see [5].

\subsection{Knowledge Representation and Annotation via Ontologies}

In $\mathcal{K}-\mathcal{M O R} \mathcal{P} \mathcal{H}$, knowledge artifacts are represented using two different (but inter-related) ontologies, namely: (i) Domain Ontology; and (ii) Knowledge Artifact Ontology (KAO). A domain ontology serves two purposes: (i) standardization of the domain-specific concepts and relations defined in the knowledge artifact ontologies; and (ii) specification of abstract knowledge links between contextually and functionally congruent knowledge components in different KAOs. A knowledge artifact ontology (KAO) serves as a lower-level ontology that captures both the structure and content of a particular knowledge artifact-such as CPG, CP 1, clinical cases etc. As a test-case, we used three location-specific (Halifax, Calgary and Winnipeg) Prostate Cancer (PC) pathways as medical knowledge artifacts, and represented them in different KAOs [1].

\subsection{Contextualizing Ontologies}

Contextualizing an ontology deals with an adaptation of its ontology model to support a local view [6]. In $\mathcal{K}-\mathcal{M O} \mathcal{R} \mathcal{P H}$, each $\mathrm{KAO}$ models the procedural knowledge of a knowledge artifact. But, by contextualizing a $\mathrm{KAO}$ we are able to provide a specialized view that models (i) a specific interpretation of its ontology concepts, and (ii) an implementation of its procedural knowledge applied in a particular context. A contextualized sub-ontology is extracted from a KAO based on the context-specific concepts, and comprises (i) instances (ii) sub-concepts,

(iii) equivalent-concepts, (iv) properties, (v) property domain and range, and (vi) assertions for the context-specific concepts.

\subsection{Morphing Constructs}

In order to represent the context under which two or more knowledge artifacts can be morphed to solve a specific problem, we defined a Morphing Construct. The morphing construct supervises the knowledge morphing process, and provides a context for determining when, where and how two or more knowledge artifacts need to be reconciled. A Morphing construct is a tuple that declares a context-specific knowledge component and its role under a defined context.

\subsection{Morphing Engine}

The $\mathcal{K}-\mathcal{M O R P H}$ morphing engine inputs the problem-context, ontologyencoded knowledge artifacts (OKAs), domain ontology, and morphing constructs. It first employs the problem-context to determine the context-specific knowledge components (i.e. contextualized sub-ontologies) from different KAOs. Based on the declarative knowledge of morphing constructs, it identifies correspondences 
between the ontology-entities (concepts, properties, and individuals) of different contextualized sub-ontologies. Based on the identified correspondences, the morphing engine employs the ontology reconciliation process that (i) aligns and then merges contextualized sub-ontologies; (ii) identifies and resolves logical inconsistencies, if present; and (iii) generates a morphed ontology, and unresolved inconsistencies in it.

\section{$4 \mathcal{K}-\mathcal{M O} \mathcal{R} \mathcal{P H}$ in Action: Morphing Clinical Pathways}

We tested the above mentioned processes in $\mathcal{K}-\mathcal{M O} \mathcal{O} \mathcal{P} \mathcal{H}$ using our $P C$ Testcase. The test-case involves (i) three medical knowledge artifacts, describing Prostate Cancer (PC) clinical pathways for three different locations (Halifax, Calgary, and Winnipeg); (ii) a problem-context; and (iii) the morphing constructs. The morphing process for the PC Test-case follows the following steps:

Step \# 1: Knowledge Representation and Annotation of PC Artifacts: The knowledge of three PC pathway artifacts are encoded into three different KAOs. Each pathway deals with four major types of tasks, namely (a) Consultation Task; (b) Non-consulation Task; (c) Referal Task; and (d) Followup Task, represented as concepts/classes in each KAO. Such tasks are supported (via properties) by other concepts such as Clinician, Decision Criteria, Frequency, Interval Duration, Investigation, Patient Condition Severity, Test Result, Followup, and Treatment.

Step \# 2: Defining a Problem-context: We defined a problem-context therapeutic decision support whereby the user is needs to morph all three PC pathways in terms of: (i) the treatments, (ii) their durations, (iii) their follow-ups, (iv) their care-settings and (v) the practitioners involved for them. The given problem-context represents context-specific interpretations, such as (a) Calgary and Halifax both share PC Clinicians; and (b) Treatments in the PC Calgary pathway can be treated as Followups in the PC Winnipeg pathway.

Step \# 3: Identifying Contextualized Sub-ontologies: Given the therapeutic decision support context, morphing constructs and three PC pathway ontologies, three contextualized sub-ontologies were generated. Each contextualized subontology was semantically validated for conceptual consistency and completeness.

Step \# 4: Context-driven Ontology Reconciliation of Sub-ontologies: The $\mathcal{K}$ $\mathcal{M O R} \mathcal{P} \mathcal{H}$ morphing engine initiated an ontology reconciliation process on the contextualized sub-ontologies, and as a result alignments were found between the classes Treatment, Followup, Frequency, Interval Duration, and Clinician. The morphing engine processes these alignments using context-axioms and PC domain-axioms to generate potential 'knowledge-links' between aligned PC treatments.

Results: Figure 2 shows the morphed knowledge for the treatment PC-Halifax: ActiveSurveillance. The morphed knowledge has determined that PC-Halifax: ActiveSurveillance can now be treated by a Primary Urologist. Based on the reconciliation of the concepts Clinician, Treatment, Followup and Interval a knowledge-base was generated in terms of a contextualized sub-ontology. 


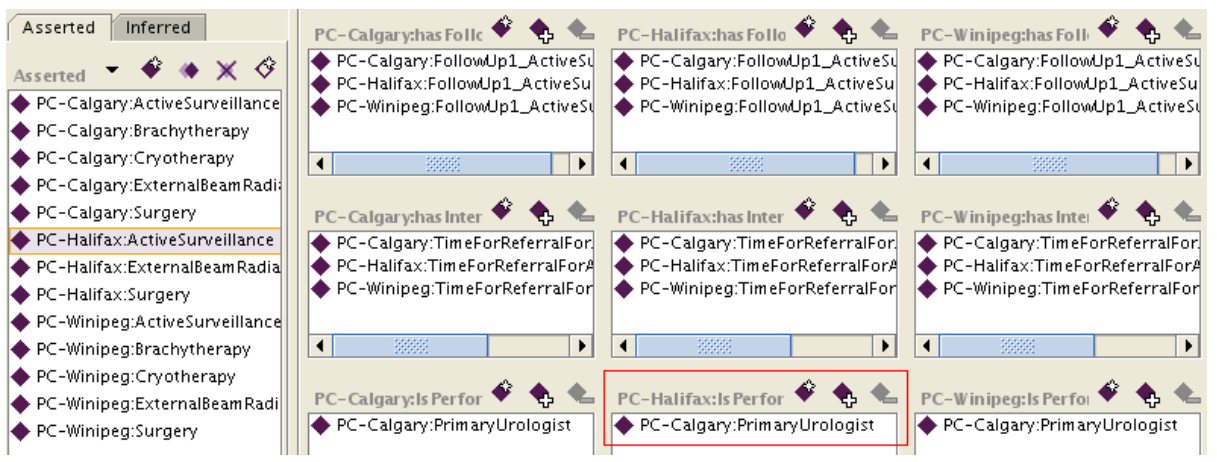

Fig. 2. PC Test-case: Morphed knowledge for PC-Halifax:ActiveSurveillance

\section{Concluding Remarks}

Clinical decision support needs a knowledge-base that can be designed from scratch or created by synthesizing existing healthcare knowledge existing in different modalities. In this paper, we presented our knowledge morphing approach that allows the systematic synthesis of multiple knowledge artifacts to develop a comprehensive knowledge-base that can be used by decision support systems. Our knowledge morphing approach ensures the semantic correctness of the morphed knowledge to the extent that it is comparable to a knowledge-base created through a knowledge engineering exercise. We showed how our approach is used to develop a unified prostate cancer clinical pathway by synthesizing three different clinical pathways.

This research is funded by a grant from Agfa Healthcare (Canada).

\section{References}

1. Abidi, S., Abidi, R., Hussain, S., Butler, L.: Ontology-based modeling and merging of institution-specific prostate cancer clinical pathways. In: Knowledge Management for Healthcare Processes Workshop at ECAI 2008, Patras, Greece, July 21-25 (2008)

2. Abidi, S.S.R.: Medical knowledge morphing: Towards the integration of medical knowledge resources. In: Computer-Based Medical Systems, June 23-24 (2005)

3. Firat, A., Madnick, S., Grosof, B.: Contextual alignment of ontologies in the eCOIN semantic interoperability framework. Inf. Tech. and Management 8(1), 47-63 (2007)

4. Robertson, D., et al.: Open knowledge: Semantic webs through peer-to peer interaction. Technical Report DIT-06-034, University of Trento, Povo, Italy (May 2006)

5. Hussain, S., Abidi, S.S.R.: K-MORPH: A semantic web based knowledge representation and context-driven morphing framework. In: Workshop on Context and Ontologies, at ECAI 2008, Patras, Greece, July 21-25 (2008)

6. Segev, A., Gal, A.: Putting things in context: A topological approach to mapping contexts to ontologies. In: Spaccapietra, S., Atzeni, P., Fages, F., Hacid, M.-S., Kifer, M., Mylopoulos, J., Pernici, B., Shvaiko, P., Trujillo, J., Zaihrayeu, I. (eds.) Journal on Data Semantics IX. LNCS, vol. 4601, pp. 113-140. Springer, Heidelberg (2007) 